\title{
Sheet Breakup and Droplet Dynamics in Gasoline Spray of Multi-Hole Port Fuel Injector
}

\author{
Kanako Nishimura*1, Dai Matsuda ${ }^{1}$, Eriko Matsumura ${ }^{2}$, Jiro Senda ${ }^{2}$ \\ ${ }^{1}$ Department of Mechanical Engineering, Doshisha University, Kyoto, Japan \\ ${ }^{2}$ Department of Science and Mechanical engineering, Doshisha University, Kyoto, Japan \\ ${ }^{*}$ Corresponding author email: ctwf0551@mail4.doshisha.ac.jp
}

\begin{abstract}
We have investigated a fuel spray from a multi-hole nozzle under gasoline port fuel injection conditions, the classification of the spray using dimensionless numbers in fluid dynamics is estimated to be in the wave like breakup region. In this study, shadowgraph photography was used to visualize the sheet breakup process of the gasoline spray. It was confirmed that the fuel formed a liquid sheet and broke up into droplets with a distribution along the axial distance from the nozzle. Also, the effects of the nozzle angle and differential injection pressure on the dynamic characteristics of fuel droplets were investigated by applying Particle Tracking Velocimetry (PTV) analysis. From these results, the direction and dispersion of droplets propagation are affected by the nozzle angle and differential pressure of injection.
\end{abstract}

\section{Keywords}

Port fuel injection, Gasoline spray, Sheet breakup, Atomization

\section{Introduction}

In internal combustion engines, a compact and simple combustion system is required to accomplish the demands from the accelerating electrification of automotive powertrains. Even more, in order to achieve strict global exhaust gas regulations, further technological advances related to fuel injection are required for gasoline engines with port fuel injection. Fuel injected to the intake port becomes vapor and flows to the cylinder. Then, some fuel impinges over the cylinder liner and back of the valve, or flows into the cylinder as droplets and impinges on the cylinder wall [1]. Previous studies have reported about fuel injection of multi-hole nozzles, but most studies focused on the wall wetting and the thickness of the deposited liquid film [2] [3], and few studies focused on the atomization process of the fuel spray [4]. However, it is important to understand the atomization process of a fuel spray to avoid the wall wetting, to control the mixture formation process and the resulting combustion process. Therefore, the purpose of this study is to reveal the atomization process of fuel spray in the port fuel injection conditions [5] [6]. In this paper, the effects of nozzle angle and differential pressure of injection on the spray atomization process and droplet dynamic characteristics after breakup were investigated.

\section{Experimental Methods and Conditions}

The experiments were conducted using plate type multi-hole nozzles (4 holes) in Figure 1. Three injectors with different nozzle angles (nozzle angle $\alpha=20,30$ and 40 deg.) were used to investigate the effect of the nozzle angle on spray formation. The nozzle diameter $\varphi d$ was $0.150 \mathrm{~mm}$ and the plate thickness $t$ was $0.1 \mathrm{~mm}$. The nozzles were prototype designed with the pitch circle diameter $D_{1}$ of $1.5 \mathrm{~mm}$ to remove the effect of interference between the sprays. Laser lighting for visualization (Cavitar Ltd.: CAVILUX Smart, wavelength $\lambda=640 \mathrm{~nm}$ ) was used as the light source, and a high-speed video camera (Photron: FASTCAM SA-Z) was used for shadowgraphs visualization of fuel spray. The frame rate was $40,000 \mathrm{fps}$. The visible range 
was $20 \mathrm{~mm} \times 7 \mathrm{~mm}$, and the spatial resolution was $18.5 \mu \mathrm{m}$. The experimental conditions are shown in Table 1. The base condition set as nozzle angle $\alpha$ was $30 \mathrm{deg}$. and differential pressure of injection $\Delta P$ was $300 \mathrm{kPa}$. Experiments were conducted in the non-evaporating conditions using nitrogen at the ambient temperature $T_{a}$ of $293 \mathrm{~K}$. The ambient pressure $P_{a}$ was $0.10 \mathrm{MPa}$ (ambient density $\left.\rho_{a}=1.15 \mathrm{~kg} / \mathrm{m}^{3}\right)$. The test fuel was iso-Octane, and the injection differential pressure $\Delta P$ was changed as 200, 250, 350, 300 and $400 \mathrm{kPa}$. Injection duration $t_{\text {inj }}$ was $10.6 \mathrm{~ms}$. The spray characteristics during the quasi-steady state were investigated after start of injection $5.0-10.0 \mathrm{~ms}$.

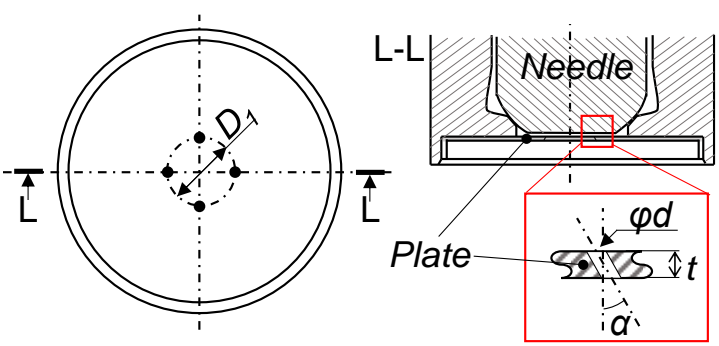

Figure 1. Nozzle specifications

Table 1 - Experimental conditions

\begin{tabular}{|c|c|c|c|c|c|}
\hline Multi Hole Nozzle $(4-\varphi 0.150 \mathrm{~mm})$ & \multicolumn{2}{|c|}{ Nozzle 1} & Nozzle 2 & \multicolumn{2}{|c|}{ Nozzle 3} \\
\hline Nozzle angle $\alpha$ [deg.] & \multicolumn{2}{|c|}{20} & 30 & \multicolumn{2}{|c|}{40} \\
\hline Test Fuel & \multicolumn{5}{|c|}{ iso-Octane (2,2,4-Trimethylpentane) } \\
\hline Differential Pressure of Injection $\Delta P[\mathrm{kPa}]$ & 200 & 250 & 300 & 350 & 400 \\
\hline Injection Duration $t_{i n j}[\mathrm{~ms}]$ & \multicolumn{5}{|c|}{10.6} \\
\hline Injection Fuel Amount $Q_{\text {inj_all }}[\mathrm{mg}]$ & 7.89 & 8.55 & 9.58 & 10.33 & 11.01 \\
\hline Fuel Temperature $T_{f}[\mathrm{~K}]$ & \multicolumn{5}{|c|}{293} \\
\hline Ambient Gas & \multicolumn{5}{|c|}{$\mathrm{N}_{2}$} \\
\hline Ambient Temperature $T_{a}[\mathrm{~K}]$ & \multicolumn{5}{|c|}{293} \\
\hline Ambient Density $\rho_{a}\left[\mathrm{~kg} / \mathrm{m}^{3}\right]$ & \multicolumn{5}{|c|}{1.15} \\
\hline Ambient Pressure $P_{a}[\mathrm{MPa}]$ & \multicolumn{5}{|c|}{0.10} \\
\hline
\end{tabular}

\section{Visualization of Sheet Breakup in Port Fuel Injection}

The breakup processes are shown in Figure 2. The dashed lines in the images indicate the nozzle angle $\alpha$. The injected fuel was formed a liquid sheet, ligaments, and then broken up into droplets. The breakup occurred with a distribution in the axial direction from nozzle. Near the nozzle exit, breakup occurs from the edge of sheet as shown in Figure 2 (a). Bag formation and breakup were also observed in Figure 2 (b). Most of the liquid sheet broke up into ligaments, and the ligaments broke up into droplets due to wave instability as shown in Figure 2 (c). Thus, the spray had spatially different processes of sheet breakup and broke up into droplets eventually.

According to research on the atomization characteristics of a plate type multi-hole nozzle, the narrower the space between the plate and the needle, the more the spray is pressured and ejected as a liquid sheet [7]. The fuel is deflected in the injector and pressed against the central axis of the injector. Therefore, a semicircular cone shaped liquid sheet is formed with edges on the outer side of the nozzle. 


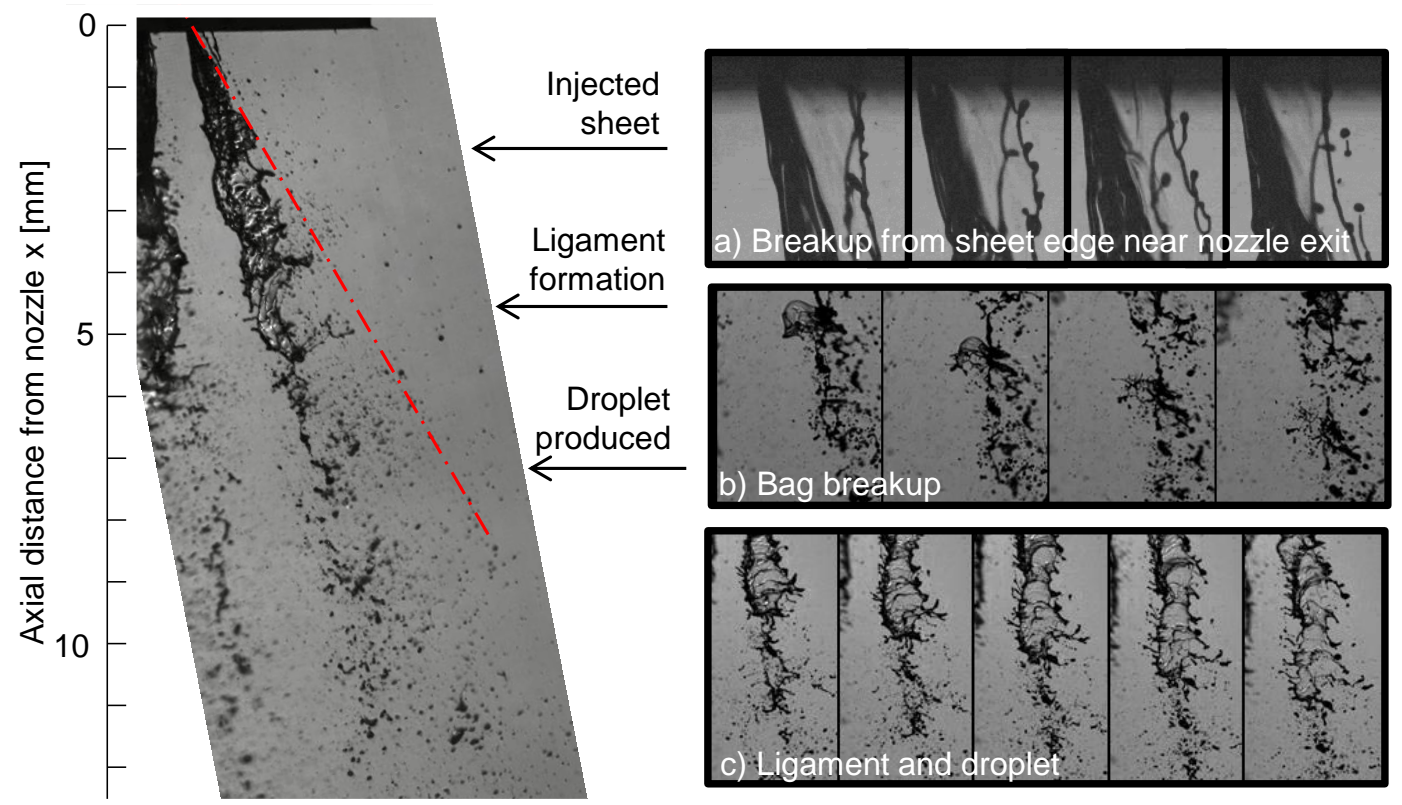

Figure 2. The processes of sheet breakup, which are injected in sheet form. There are ligament formation and droplet production. Some breakup patterns; (a) Breakup from edge of liquid sheet near the nozzle exit, (b) Liquid sheet forms like bag partially, and it breaks up into small droplets, (c) Ligament formation and its breakup into droplets.

\section{Droplets Dispersion Characteristics}

In order to investigate the dispersion characteristics of droplets in detail, the distributions of the droplet dispersion angle were calculated. Figure 3 shows droplet spatial distribution of the fuel spray with the different nozzle angle. The dashed line in the image indicates the nozzle angle $\alpha$. The dynamic characteristics of the droplets were obtained by PTV analysis during the quasi-steady period. In PTV, the droplet size and location are measured for the first image taken at time $t$ and the second image taken at time $t+\Delta t$. The region around the droplet obtained from the first image is searched for in the second image, and those droplets with a size ratio of $90-110 \%$ are identified as the same droplet and its velocity and angle are measured from the distance it moved. Figure 4 shows the number and mass frequency distributions of fuel spray droplet dispersion angles at each nozzle angle. The frequency distributions are normalized by the total number of the observed fuel droplets with dispersion angles between -10deg. and 50deg.. The fuel spray droplets are dispersed at an angle smaller than the nozzle angle in all conditions. Figure $\mathbf{5}$ shows the discharge coefficients during the quasi-steady state of the injection, calculated from the injection amount. The discharge coefficient decreased with the increase of the nozzle angle. Larger nozzle angles produced more separation at the nozzle inlet [8]. The fuel that enters the nozzle after separating at the nozzle inlet is more pressured toward the central axis of the injector as the nozzle angle is larger, so the discharge coefficient changes depending on the nozzle angle. It is assumed that the liquid sheet formed after injection has a different shape at each nozzle angle.

In the case of the nozzle angle at 20 deg., there is a bias in the droplet dispersion angle distribution. This is due to the liquid sheet forming a semicircular cone, with the larger droplets dispersed on the central axis of the injector, and the smaller droplets dispersed on the edge of the sheet. The difference in the distribution became smaller as the nozzle angle increased, because smaller droplets and higher dispersion were produced. The frequency distribution indicates that the larger droplets are dispersed in the direction of the larger mass frequency compared to the number frequency, while the smaller droplets are dispersed in the direction of the smaller mass frequency compared to the number frequency. When the mass frequency is smaller than the number frequency, smaller droplets are dispersed in the direction. 


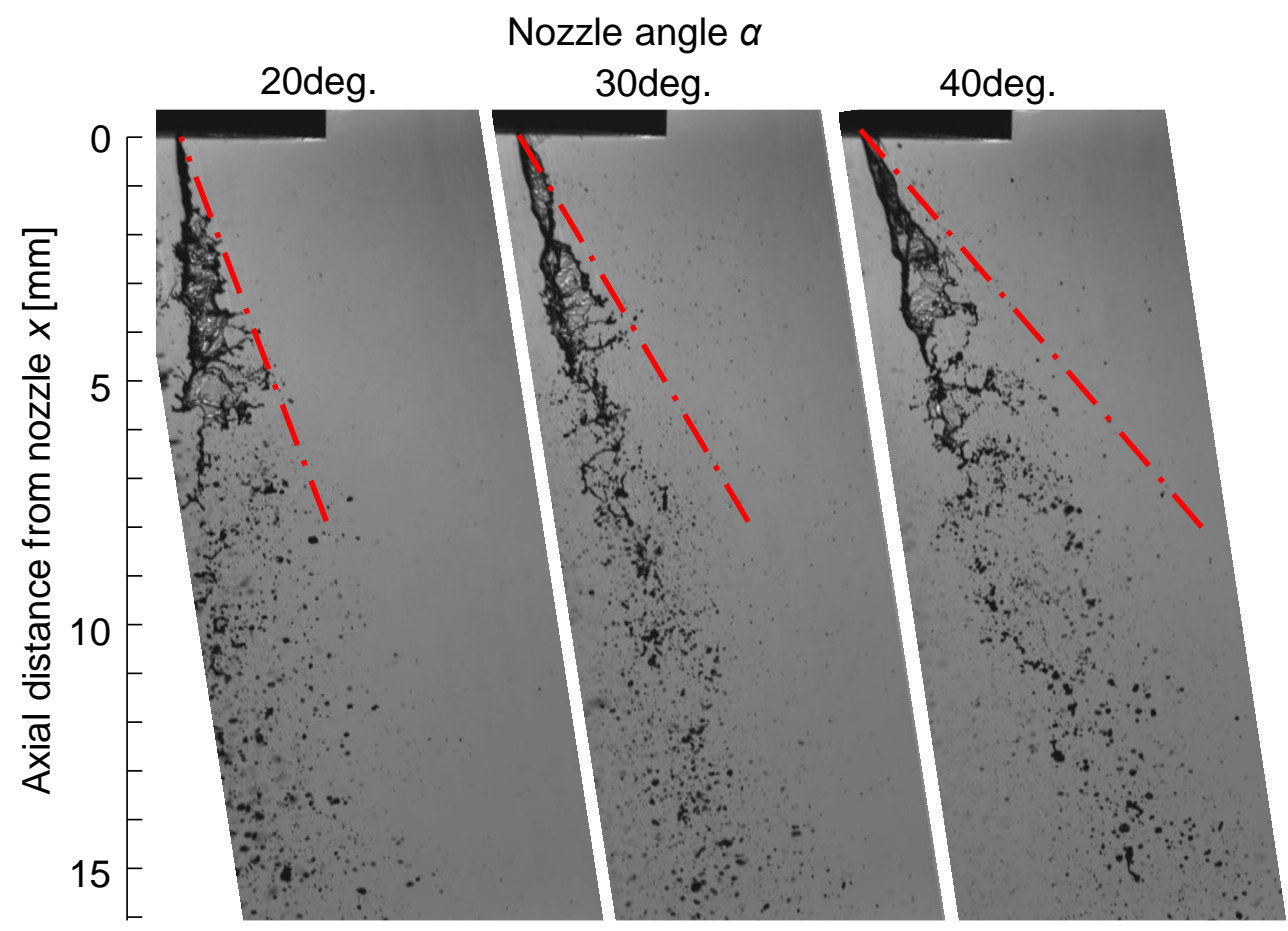

Figure 3. Droplet spatial distribution of the fuel spray ASOI $7.0 \mathrm{~ms}$ with different nozzle angle at $\Delta P=300 \mathrm{kPa}$

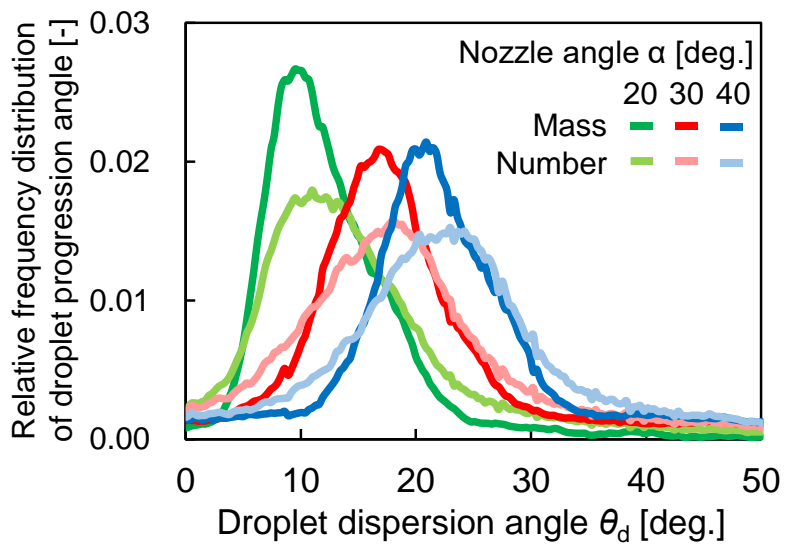

Figure 4. Relative frequency distribution of droplet dispersion angle at $\Delta P=300 \mathrm{kPa}$

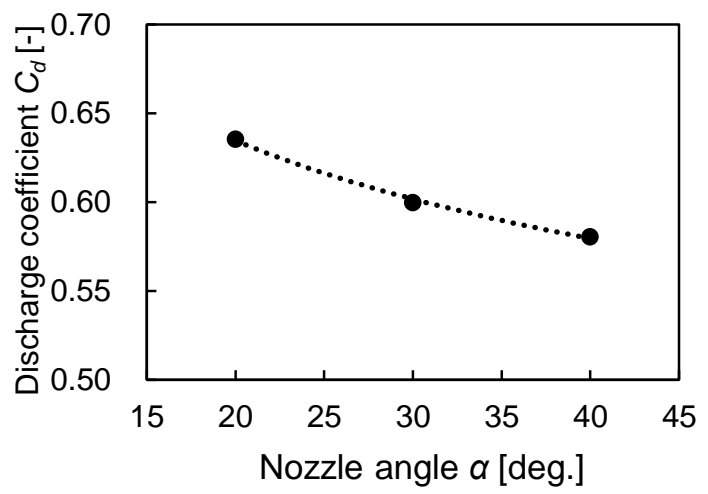

Figure 5. Discharge coefficient v.s. nozzle angle at $\Delta P=300 \mathrm{kPa}$

Figure 6 shows the mode angle of mass frequency and number frequency as characteristic values of distribution, and the standard deviation of mass frequency distribution as characteristic values of dispersion. The results also show that the standard deviation increases with the nozzle angle, indicating that the spray is dispersed.

The effects of differential pressure of injection were investigated in the same procedure in Figure 7. The mode angle of mass frequency and number frequency, and the standard deviation at each differential pressure of injection are shown in the figure. It was found that the dispersion angle increases as the differential pressure of injection increases. The angle of the maximum number frequency increased and the angle of the maximum mass frequency decreased with the increase of the differential pressure of injection. The angle of the maximum number frequency increased with the higher differential pressure of injection. This is due to the increase in the number of small droplets dispersed at larger angles. It is also found that the decrease in the angle of maximum mass frequency with increasing injection pressure. It 
is caused by the effect that the mainstream liquid sheet is pushed to the inner side of the spray by the higher pressure. The higher injection velocity maintains the velocity component in the streamwise direction between the plate and the needle. The large droplets generated from the liquid sheet at the center axis of the nozzle maintain their dispersal direction due to their high momentum, while the small droplets generated from the outer liquid sheet cannot maintain their original dispersal direction due to the drag force.

These will be discussed in terms of the exponent of each parameter. As a spray direction characteristics value, the deviation of the droplet dispersion angle from the nozzle angle is defined as the difference of droplet dispersion angle from the nozzle angle $\alpha$ - $\theta_{d \text {. The angles }}$ of maximum mass frequency are used as a representative of the droplet dispersion angle $\theta_{d}$ in the index correlations. As a result, the exponent of the deviation for each experimental parameter were 0.84 for the nozzle angle and 0.32 for the differential pressure of injection. The exponent of the standard deviation, which is a characteristic value of dispersion, were 0.56 for the nozzle angle and 1.31 for the differential pressure of injection. Thus, the spray direction and its dispersion are different due to the film formation and breakup process.

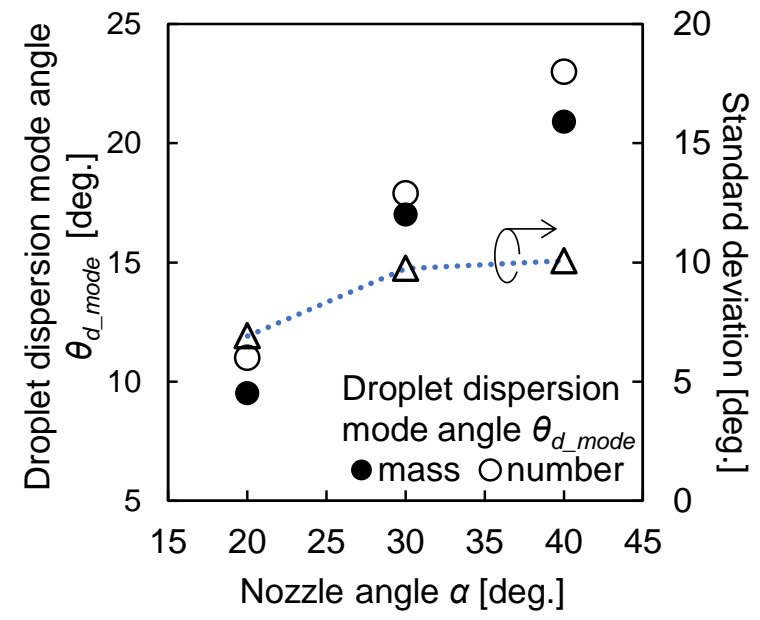

Figure 6. Droplet dispersion mode angle and standard deviation v.s. nozzle angle at $\Delta P=300 \mathrm{kPa}$

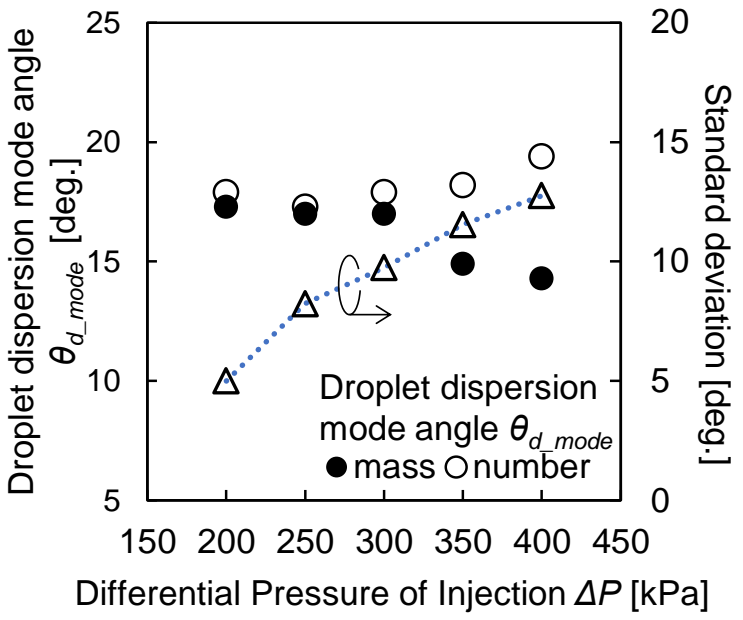

Figure 7. Droplet dispersion angle and standard deviation v.s. differential pressure of injection $\Delta P$ at $\alpha=30 \mathrm{deg}$.

\section{Atomization Characteristics and Weber Number of Droplets}

The behavior of droplet breakup and wall impingement is characterized by non-dimensional numbers; the gas Weber number $W e_{g}$, which takes the density of surrounding gas as the density term, is used to represent the breakup of fuel droplet [9] [10], and the liquid Weber number $W e$, which takes the liquid density as the density term, is used to classify the impingement of droplet into wall and liquid film [11] [12]. Here, Weber number $W e_{g}$ and $W e_{l}$ are defined as follows:

$$
\begin{aligned}
& W e_{g}=\rho_{a} U_{r}^{2} d / \sigma \\
& W e_{l}=\rho_{I} U_{n}^{2} d / \sigma
\end{aligned}
$$

Where, $\rho_{a}$ and $\rho_{l}$ are density of gas and liquid, is relative velocity between gas and liquid, is normal velocity, is droplet diameter, and is surface tension between fuel and ambient gas.

Thus, we investigated the diameter and velocity of fuel droplets by PTV analysis. In this study, the droplets measured by PTV are considered as the total droplets in the spray, and the distributions of droplet size and Weber number are calculated. Figure $\mathbf{8}$ shows the mass 
frequency distribution of droplet sizes during the quasi-steady state period. The distribution shifted to smaller droplet sizes with higher injection pressure. The percentage of droplets with diameter larger than $200 \mu \mathrm{m}$ was $7.3 \%$ at $200 \mathrm{kPa}, 6.4 \%$ at $300 \mathrm{kPa}$, and $3.4 \%$ at $400 \mathrm{kPa}$, indicating that the ratio of large droplets decreased with increasing differential pressure of injection.

The mass frequency distribution of the Weber number during the quasi-steady period is shown in Figure 9. The distribution was weighted by multiplying the frequency by $d^{3}$ to obtain the mass distribution. The distribution shifts to the higher Weber number with the increase of the injection differential pressure. According to the classification of breakup modes of single droplets by Hsiang et al., droplets with the gas Weber number We $e_{g}$ less than 11 are classified as vibration breakup, where little breakup occurs [10]. The number of droplets with the gas Weber number $W e_{g}$ more than 11 is less than $2.5 \%$ of the total droplets in all conditions. It is suggested that the secondary breakup, in which droplets break up into further smaller droplets, does not occur in the port fuel injection conditions. As for the liquid Weber number $W e_{l}$, which classifies the behavior of wall impingement. In this experiments, the obtained distribution is for droplets observed in the entire spray area, so the liquid Weber number at wall impingement decreases due to velocity slow down.

Figure 10 shows the Sauter mean diameter (SMD) at each nozzle angle (nozzle angle $\alpha$ : 20, 30, 40 deg.) when the differential pressure of injection was changed. SMD were calculated from the droplet size distribution mentioned above. SMD decreased with increasing differential pressure of injection. The effect of injection pressure decreased as the nozzle angle increased. These results can be explained that the number of large droplets decreases with the increase of nozzle angle and differential pressure of injection. Figure 11 shows the mass average of the Weber number. It was calculated as the median of the mass distribution of the Weber number in Figure 9. The median Weber number increased with increasing pressure. Its exponent for the differential pressure of injection was 0.42 at 20 deg., 0.58 at 30 degrees, and 0.69 at 40 degrees, and the index value increased with the increase of the nozzle angle. This is thought to be caused by the decrease in the number of large droplets due to the increase in the nozzle angle and the injection pressure. The smaller the droplet diameter, the smaller the droplet velocity after breakup. However, the larger the nozzle angle is, the smaller the number of larger droplets becomes, and the exponent of the SMD for the differential pressure of injection becomes smaller. Therefore, the mean value of the Weber number decreases with the increase of the nozzle angle, and the exponent of the Weber number against the differential pressure of injection increases with the increase of the nozzle angle.

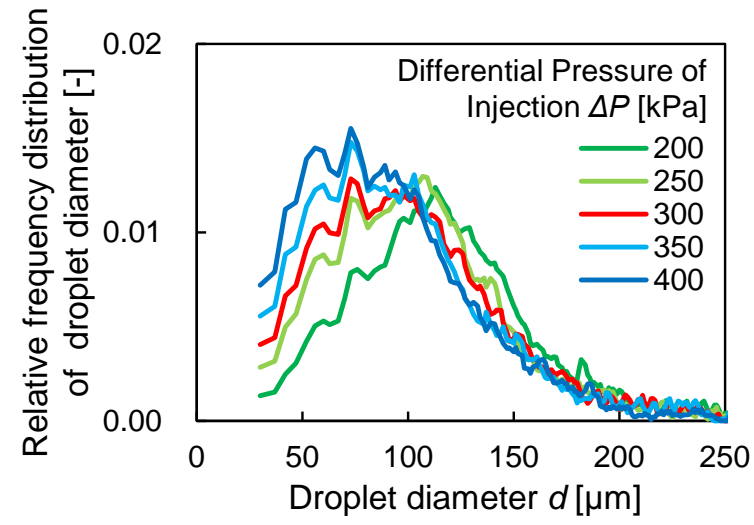

Figure 8. Relative frequency mass distribution of Droplet diameter at $\alpha$ : $30 \mathrm{deg}$.

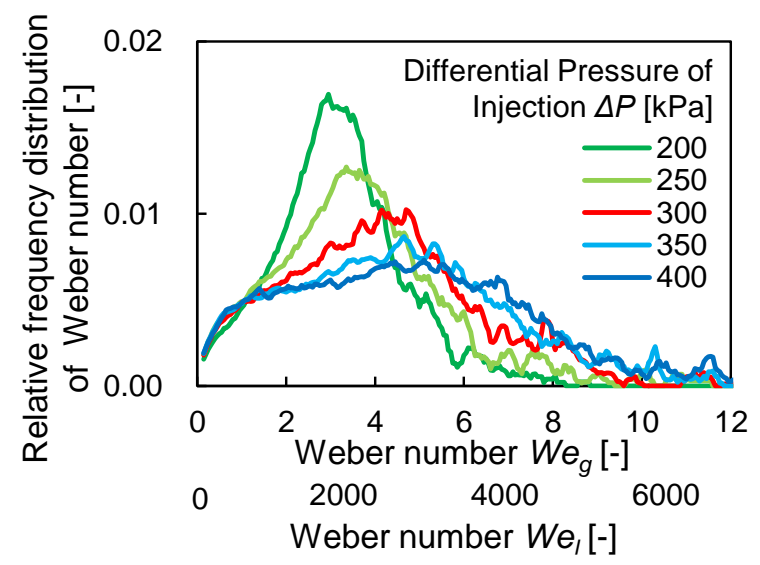

Figure 9. Relative frequency mass distribution of Weber number $W_{g}, W e_{l}$ at $\alpha: 30 \mathrm{deg}$. 


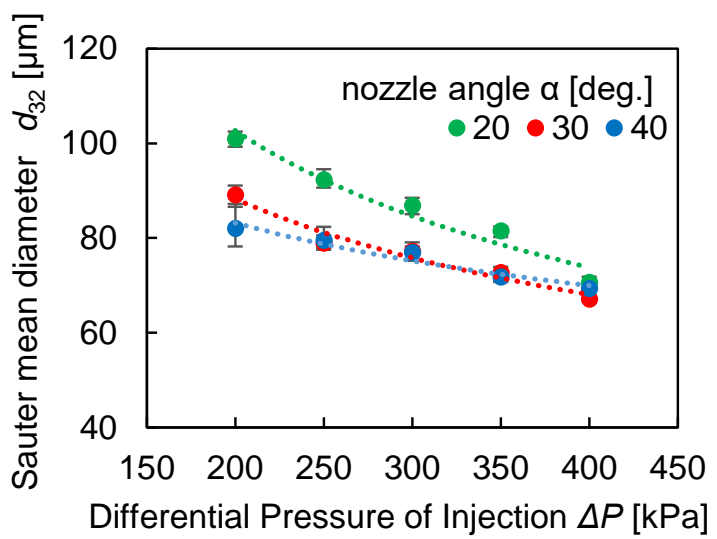

Figure 10. Sauter mean diameter with differential pressure of injection at different nozzle angle conditions.

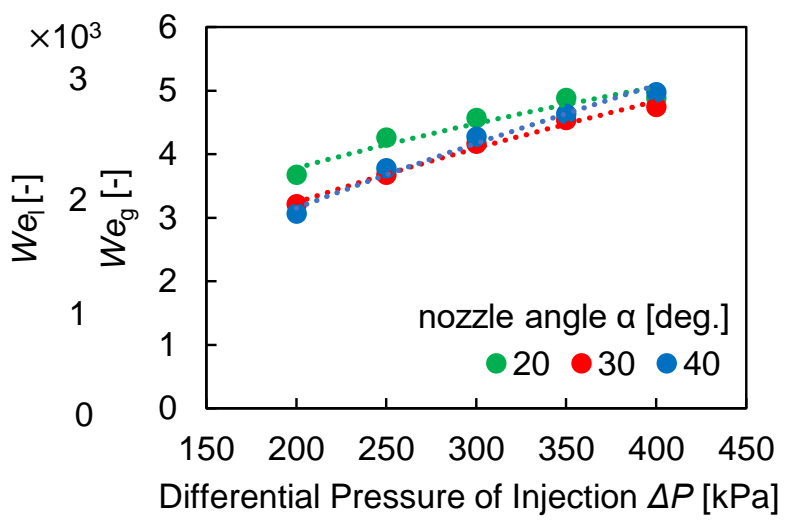

Figure 11. Mass average of Weber number with differential pressure of injection at different nozzle angle conditions.

\section{Conclusions}

In this paper, the fuel spray from a multi-hole nozzle for port fuel injection was investigated by visualizing the sheet breakup process and analyzing the droplet dynamic characteristics by PTV analysis.

The formation of liquid sheets near the nozzle exit was observed in the fuel injected from the multi-hole nozzle. The fuel droplets dispersed with the distribution centered at an angle smaller than the nozzle angle. Large droplets were distributed the direction of the sheet injection, while small droplets were spread over a wide range. The direction and dispersion of droplets propagation are affected by the nozzle angle and differential pressure of injection. Regarding the atomization characteristics, SMD decreased and Weber number increased with increasing differential pressure of injection. The difference with different nozzle angle got smaller as the differential pressure of injection increased.

\section{Acknowledgments}

The authors gratefully acknowledge Mr. Takeda of Aisan Industry Co., Ltd. for producing the prototype nozzles.

\section{Nomenclature}

$\alpha \quad$ nozzle angle [deg.]

$C_{d} \quad$ discharge coefficient [-]

$d \quad$ droplet diameter $[\mu \mathrm{m}]$

$d_{32} \quad$ Sauter mean diameter $[\mu \mathrm{m}]$

$D_{1} \quad$ pitch circle diameter [mm]

$\Phi d \quad$ nozzle diameter [mm]

$\Delta P \quad$ differential pressure of injection [ $\mathrm{kPa}]$

$Q_{\text {inj_all }}$ Injection amount with all nozzles (4 holes) [mg]

$t \quad$ plate thickness [mm]

$t_{i n j} \quad$ injection duration [ms]

$T_{\mathrm{a}} \quad$ ambient temperature $[\mathrm{K}]$

$T_{f} \quad$ fuel temperature $[\mathrm{K}]$

$U_{n} \quad$ velocity component of impinging droplet in normal direction to the wall [m/s]

$U_{r} \quad$ relative velocity between fuel droplet and ambient gas $[\mathrm{m} / \mathrm{s}]$

$\rho_{a} \quad$ ambient density $\left[\mathrm{kg} / \mathrm{m}^{3}\right]$

$\rho_{l} \quad$ liquid density $\left[\mathrm{kg} / \mathrm{m}^{3}\right]$ 
Weg gas Weber number [-]

$W e_{1} \quad$ liquid Weber number [-]

$x \quad$ axial distance from nozzle [mm]

$\theta_{d} \quad$ droplet dispersion angle [deg.]

$\theta_{d \_ \text {mode }}$ droplet dispersion mode angle [deg.]

\section{References}

[1] Takeda, K., Yaegashi, T., Sekiguchi, K., Saito, K., Imatake, N., 1995, SAE Technical Paper, No. 950074.

[2] Moriyoshi, Y., Yamada, T., Kuboyama, T., Sato, G., Dec. 17-18. 2012, 21st ILASS-Japan Symposium.

[3] Takahasi, Y., Nakase, Y., Sato, T., Kubota, M., 2008, Transactions of Society of Automotive Engineers of Japan, 39 (3), pp.131-136.

[4] Nagaoka, M., 1998, R\&D Review of Toyota Central R\&D Laboratory, 33 (2), pp.23-35.

[5] Nishimura, K., Matsuda, D., Matsumura, E., Senda, J., 2021, Transactions of Society of Automotive Engineers of Japan, in review.

[6] Nishimura, K., Matsuda, D., Matsumura, E., Senda, J., 2021, Transactions of Society of Automotive Engineers of Japan, in review.

[7] Suzuki, T., Tani, Y., Tokutomi, H., 2004, journal of the ILASS-Japan, 13 (41), pp.136-147.

[8] Yano, H., Matsuura, M., Murakami, S., Haibara, T., Matsumura, E., Senda, J., 2018, Transactions of the Society of Automotive Engineers of Japan, 49 (1), pp.18-23.

[9] Hinze, J. O., 1955, American Institute of Chemical Engineers Journal,1 (3), pp.289-295.

[10] Hsiang, L. P. and Faeth, G. M., 1992, Int. J. Multiphase Flow, 18 (5), pp.635-652.

[11] Senda, J., Matsuda, T., 2003, journal of the ILASS-Japan, 12(39), pp.38-53.

[12] Naber, J. D., Reitz, R. D., 1988, SAE Technical Paper, No. 880107. 